\title{
Spatiotemporal Soliton Attractor in Multimode Graded-index Fibers
}

\author{
Mario Ferraro ${ }^{1,}{ }^{*}$, Mario Zitelli ${ }^{1}$, Fabio Mangini ${ }^{2}$, and Stefan Wabnitz ${ }^{1}$ \\ ${ }^{1}$ Department of Information Engineering, Electronics and Telecommunications (DIET), Sapienza \\ University of Rome, Via Eudossiana 18, 00184 Rome, Italy \\ 2 Department of Information Engineering (DII), University of Brescia, Via Branze 38, 25123 Brescia, Italy \\ *mario.ferraro@uniroma1.it
}

There is recently a considerable interest in nonlinear optical effects in multimode fibers (MMFs). Here we present the experimental evidence, supported by numerical simulations, that an initially excited multimode femtosecond soliton initially composed by few low-order transverse modes of a graded-index (GRIN) MMF irreversibly decays into a singlemode soliton carried by the fundamental mode of the MMF. Once formed, the singlemode soliton propagates stably over fiber lengths of up to $1 \mathrm{~km}$, which corresponds to a record transmission distance, in a MMF, of 4600 modal dispersion distances, and 5600 chromatic dispersion lengths. This effect is of particular importance for technological applications, since it reveals that nonlinearity can counteract the effects of modal dispersion and random mode coupling, and enables a stable transport of high-spatial-quality beams over long distances by means of large area (when compared to singlemode fibers) MMFs. As a side aspect, our results invalidate theoretical predictions based on variational approaches, since the beam shape substantially evolves along the fiber, in such a way that the initial beam profile is not maintained [1-3].
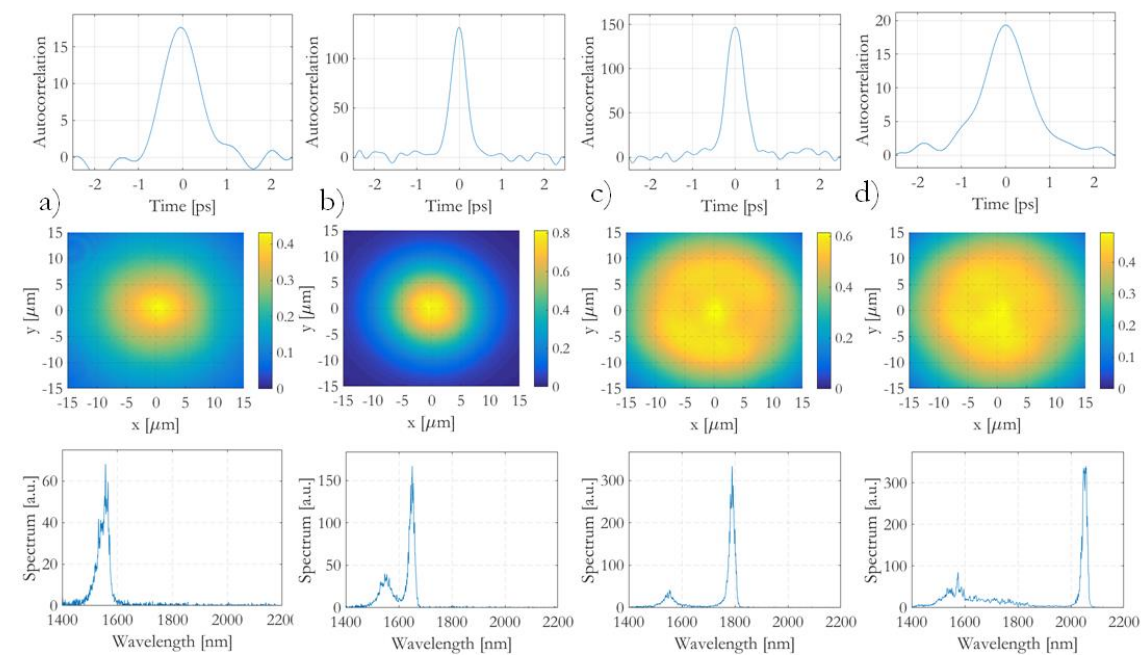

Fig. 1 Measured autocorrelation traces (top row), beam near-field (central row), and spectra (bottom row) in four different regimes: (a) pre-soliton at $22 \mathrm{~kW}$, (b) soliton at $29 \mathrm{~kW}$, (c) soliton with SSFS at $37 \mathrm{~kW}$ and multiple soliton generation at $109 \mathrm{~kW}$.

Fig. 1 shows an example of measured autocorrelation traces, beam near-field and spectra (bottom row) after $120 \mathrm{~m}$ of fiber transmission, for a $70 \mathrm{fs}$ input pulse at $1550 \mathrm{~nm}$ and $30 \mu \mathrm{m}$ input diameter. Four regimes (presolitonic, solitonic, solitonic with Raman soliton self-frequency-shift (SSFS) and multiple soliton generation) are reported. Experimental data confirm the generation of a pulse with minimum duration at the soliton power (25-30 $\mathrm{kW}$ input peak power). Output spectra show sech-shaped lobes, subject to SSFS shift up to the wavelength of linear absorption, of about $2100 \mathrm{~nm}$. As can be seen, the output beam reaches both its minimum waist and pulsewidth at the same power value of $29 \mathrm{~kW}$; the beam waist reduces down to $8.5 \mu \mathrm{m}$, close to the $7.7 \mu \mathrm{m}$ theoretical value for the fundamental mode beam in a GRIN fiber. This confirms that a substantially fundamental mode has survived at the soliton power; the minimum pulsewidth was $250 \mathrm{fs}$ at $120 \mathrm{~m}$. For higher input peak powers $(110 \mathrm{~kW}$ and $190 \mathrm{~kW})$, a second and third soliton are observed at the MMF output: each of them experiences a beam waist reduction. Multiple solitons are generated from a common fission process after a few meters of propagation; the first soliton eventually experiences Raman SSFS up to $2100 \mathrm{~nm}$ and it is absorbed; the second and third solitons shift at higher powers, so that they can be observed at the MMF output. We anticipate that our results will have significant impact also on spatiotemporal mode-locking based on MMF lasers. We acknowledge support from EU Horizon2020 ERC Grants No. 874596 and No. 740355.

[1] A. S. Ahsan and G. P. Agrawal, "Graded-index solitons in multimode fibers," Opt. Lett. 43, 3345-3348 (2018).

[2] S.-S. Yu, C.-H. Chien, Y. Lai, and J. Wang, "Spatio-temporal solitary pulses in graded-index materials with Kerr nonlinearity," Opt. Commun. 119, 167-170 (1995).

[3] S. Raghavan and G. P. Agrawal, "Spatiotemporal solitons in inhomogeneous nonlinear media," Opt. Commun. 180, 377 - 382 (2000). 